\title{
Asociación entre el estado nutricional según índice de masa corporal y deterioro cognitivo en adultos mayores del Centro Médico Naval del Perú, 2010-2015
}

\section{Association between nutritional status determined using body mass index and cognitive impairment in elderly adults attending at Centro Medico Naval del Peru, 2010-2015}

Correspondencia Lorena Bazalar Silva Calle Las Trinitarias $\mathrm{N}^{\circ} 115$, Salamanca, Lima-Perú. Teléfono: 5114341232 baza.silva.lore@gmail.com

Recibido: 22/01/2019

Arbitrado por pares

Aprobado: 06/03/2019

Citar como: Bazalar-Silva L, Runzer-Colmenares FM, Parodi JF. Asociación entre el estado nutricional según índice de masa corporal y deterioro cognitivo en adultos mayores del Centro Médico Naval del Perú, 2010-2015. Acta Med Peru. 2019;36(1):5-10
Lorena Bazalar-Silva ${ }^{1 a}$, Fernando M. Runzer-Colmenares ${ }^{1,2 b}$, José F. Parodi²b

1 Universidad Científica del Sur. Lima, Perú

2 Centro de Investigación del Envejecimiento, Universidad de San Martín de Porres. Lima, Perú.

a Estudiante de Medicina, b médico geriatra

\section{RESUMEN}

Objetivo: Evaluar la relación entre el estado nutricional y el deterioro cognitivo en adultos mayores atendidos en el Centro Médico Naval "Cirujano Mayor Santiago Távara" en el periodo 2010-2015. Materiales y métodos: Estudio cuantitativo, observacional, analítico, retrospectivo, transversal. Se incluyeron 1594 pacientes adultos mayores que cumplieron los criterios de selección. La evaluación del deterioro cognitivo fue mediante la prueba MoCA, MMSE, y el cuestionario funcional de Pfeiffer. Se utilizó el software estadístico STATA. Mediante regresión lineal se analizó el deterior cognitivo según índice de masa corporal. Resultados: La edad promedio fue de $78,4 \pm 8,5$ años. Hubo más varones (59\%) que mujeres (41\%). Cerca del $25 \%$ tenía bajo peso. El promedio de puntaje MoCA y MMSE fue significativamente menor en los participantes con bajo peso, mientras que se obtuvo una mediana significativamente más alta en la prueba de Pfeiffer para aquellos con bajo peso. Conclusión: Se encontró relación significativa entre el bajo peso y el deterioro cognitivo evaluado por MoCA, MMSE y Pfeiffer en adultos mayores incluidos en el estudio.

Palabras clave: Estado nutricional; Pruebas de estado mental y demencia; Demencia; Anciano (fuente: DeCS BIREME). 


\section{ABSTRACT}

Objective: To evaluate the relationship between nutritional status and cognitive impairment in elderly adults attending the 'Cirujano Mayor Santiago Tavara' Peruvian Navy Medical Center between 2010 and 2015. Materials and methods: This is an observational, analytical, retrospective, and cross-sectional study. We included 1594 elderly patients who met the selection criteria. The evaluation of cognitive impairment was performed using the following instruments: MoCA, MMSE, and the Pfeiffer questionnaire. STATA statistical software was used, and linear regression models were also used for analyzing cognitive impairment according to the body mass index. Results: Subjects' average age was $78.4 \pm 8.5$ years. There were more males (59\%) than females (41\%). About $25 \%$ were underweight. Both MoCA and MMSE mean scores were significantly lower in those participants with low weight, while significantly higher median values were obtained in the Pfeiffer test in low weight subjects $(p=0.0001)$. Conclusion: There is a significant relationship between low weight and cognitive impairment according to MoCA, MMSE, and Pfeiffer instruments in elderly adults included in this study.

Keywords: Nutritional status; Mental status and dementia tests; Dementia; Aged (source: MeSH NLM).

\section{INTRODUCCIÓN}

Alrededor del mundo, el porcentaje de adultos mayores de 60 años se incrementará del $12 \%$ al $22 \%$ entre los años 2015 al $2050^{[1]}$. En el Perú se estima que, para el año 2025, la población geriátrica se incrementará de tres a 4,3 millones de personas ${ }^{[2]}$. Este grupo etario se caracteriza por presentar cambios morfológicos cerebrales normales asociados a actividades funcionales a nivel cognitivo ${ }^{[3]}$. Pasados los 60 años, hay una evidente disminución en la capacidad de memoria, elocuencia expresiva y habilidad matemática ${ }^{[4]}$; sin embargo, los daños cognitivos están sujetos en su mayoría a factores fisiológicos, ambientales e interpersonales ${ }^{[5]}$.

Por otro lado, los cambios asociados al envejecimiento también afectan el estado nutricional, ya sea por el escaso consumo de macro y micronutrientes, la presencia de deterioro cognitivo, modificaciones en la ingesta de medicamentos o situaciones psicosociales y económicas ${ }^{[6-8]}$.

El aspecto nutricional tiene un papel fundamental en el buen funcionamiento cerebral ${ }^{[9]}$. Diversas investigaciones encontraron que un estado de malnutrición está asociado a una mayor prevalencia de déficit cognitivo o deterioro cognitivo grave ${ }^{[10,11]}$. A nivel nacional, Guevara demostró que el estado nutricional del adulto mayor se asocia significativamente con el estado cognitivo y que hay un incremento del deterioro cognitivo condicionado a la obesidad ${ }^{[12]}$.

Con el envejecimiento, el cerebro experimenta cambios morfológicamente normales que, dependiendo de la plasticidad cerebral y variabilidad interpersonal, pueden llegar a provocar alteraciones cognitivas. El deterioro cognitivo puede dividirse en leve (déficit en las tareas ocupacionales y sociales), moderado (deficiencia en realización de tareas complejas en relación a las áreas individuales sociales y económicas) y severo (reducción en la habilidad para realizar tareas diarias, retención de datos y desorientación en tiempo-espacio) ${ }^{[13]}$.

Para evaluar el deterioro cognitivo es necesario un examen neurológico-psicológico. Este análisis brinda datos para una futura valoración del deterioro y su seguimiento ${ }^{[12]}$. Hoy en día están disponibles múltiples test que apoyan el diagnóstico y evalúan la severidad de la disminución de la capacidad cognitiva en la población adulta mayor como el Montreal Cognitive Assessment (MoCA) ${ }^{[14,15]}$, el Mini-Mental State Examination (MMSE) ${ }^{[16,17]}$ y el Cuestionario de Pfeiffer ${ }^{[17,18]}$. No obstante, la aplicación única de estas pruebas no es suficiente para realizar el diagnóstico, sino que debe, además, hacerse una evaluación clínica completa junto con los criterios de deterioro cognitivo presentes en el DSM-5 (del inglés: Diagnostic and Statistical Manual of Mental Disorders, Fifth edition).

En cuanto a la valoración del estado nutricional en la población adulta mayor, existen varias herramientas que permiten la detección de malnutrición ${ }^{[19]}$. El índice de masa corporal (IMC), parámetro que estima el peso sobre la talla al cuadrado, es uno de los más empleados por su simplicidad.

El propósito de nuestra investigación es evaluar la relación entre el estado nutricional y el deterioro cognitivo en adultos mayores atendidos en el Centro Médico Naval "Cirujano Mayor Santiago Távara" en el periodo 2010-2015, con el fin de afianzar el conocimiento de profesionales de la salud con datos propios de nuestra región, y de esta manera, se promueva una cultura de prevención de la malnutrición (obesidad y delgadez) y promoción de la salud mental entre los adultos mayores.

\section{MATERIALES Y MÉTODOS}

\section{Tipo de estudio}

Estudio observacional, transversal, retrospectivo, con análisis secundario de una base de datos.

\section{Población y muestra}

Se incluyeron a participantes de un estudio previo ${ }^{[20]}$ realizado en adultos mayores atendidos ambulatoriamente en el Centro Médico Naval "Cirujano Mayor Santiago Távara" en el periodo 2010-2015. Dicho hospital atiende a los militares asimilados a la Marina de Guerra del Perú, tanto en actividad como en situación de retiro y a sus familiares directos. Dicho hospital cuenta con un servicio de Geriatría, locación del estudio original. 


\section{Criterios de selección}

Se incluyó a todos los atendidos en el hospital, atendidos en el servicio de Geriatría, con 60 años a más y procedentes de Lima y Callao, Perú. En su mayoría fueron hombres y personal militar en retiro, aunque también se incluyó a esposas o padres del personal militar.

Se excluyeron a aquellos pacientes con demencia establecida, depresión, problemas visuales severos o auditivos $u$ otros problemas físicos, así como aquellos consumidores de fármacos disminuyeran el rendimiento cognitivo.

\section{Variables}

Se evaluaron las características sociodemográficas (edad, sexo y nivel educativo). También el estado nutricional según IMC [21], con la atingencia de usar puntos de corte diferentes por tratarse de una población adulta mayor: bajo peso $\left(\mathrm{IMC}<22 \mathrm{~kg} / \mathrm{m}^{2}\right)$, eutrófico (IMC entre 22 y $27 \mathrm{~kg} / \mathrm{m}^{2}$ ), sobrepeso (IMC entre 27 y $\left.32 \mathrm{~kg} / \mathrm{m}^{2}\right)$ y obesidad $\left(\mathrm{IMC}>32 \mathrm{~kg} / \mathrm{m}^{2}\right)^{[22]}$. A pesar de que el IMC no es el mejor parámetro para evaluar estado nutricional, se lo utilizó con la finalidad de tener una muestra mayor. La información de peso y talla se tomó de la ficha de evaluación del consultorio externo.

El deterioro cognitivo ${ }^{[16,17]}$ se evaluó mediante tres instrumentos: 1) la prueba MoCA, que consta de trece tareas que cubren siete dominios, con una puntuación máxima de 30 , en donde un resultado menor a 26 indica deterioro cognitivo ${ }^{[15,23]}$; 2) el MMSE, que consta de cinco subsecciones, con puntuaciones máximas posibles en cada subsección (10, 3, 5, 3 y 9), pudiendo variar el resultado de 0 a 30, en donde un puntaje más alto indica un mejor rendimiento cognitivo ${ }^{[17,24]}$, y 3 ) el cuestionario de Pfeiffer, que consiste en diez preguntas, indica sospecha deterioro cognitivo cuando se comenten tres o más errores en personas que sepan leer y escribir y cuatro o más en aquellas que no ${ }^{[16,17,25]}$. Para las comorbilidades, se obtuvo el dato de la historia clínica de los participantes.

\section{Análisis estadístico}

Los datos fueron procesados en el programa STATA versión 14. Se construyeron tres modelos de regresión lineal para determinar la asociación entre IMC con trastorno neurocognitivo según las tres escalas utilizadas en el estudio. Se escogieron las técnicas de análisis estadístico para las variables numéricas según su distribución en el histograma; así, para analizar variables con distribución normal en las cuatro categorías de IMC usamos ANOVA y para el caso de las variables con distribución no normal, Kruskall Wallis. Para los tres casos se calcularon los coeficientes $\beta$ en modelos ajustados según las variables significativas en el análisis bivariado.

\section{Aspectos éticos}

La información utilizada en el estudio se obtuvo con el consentimiento de las respectivas autoridades de la Universidad Científica del Sur y Centro Médico Naval (aprobaciones institucionales y éticas).

\section{RESULTADOS}

Se consideró estudiar a toda la población ( $n=1896)$, sin embargo, se excluyeron a 302 participantes porque tenían dependencia funcional severa, lo que impidió medirles el peso y la talla y, por consiguiente, el IMC. En todos los casos, no hubo datos faltantes superiores al $10 \%$ de la muestra total. La muestra final estuvo conformada por 1594 adultos mayores.

Respecto a las características sociodemográficas, el promedio de edad fue de $78,4 \pm 8,5$ años, la proporción de varones (59,4\%) fue superior a la de mujeres $(40,6 \%)$ y el $74,9 \%$ estudió por al menos 11 años, es decir, o no culminó la educación secundaria 0 apenas lo hizo (Tabla 1).

Tabla 1. Características de los pacientes del estudio $(n=1594) *$

\begin{tabular}{lc}
\multicolumn{1}{c}{ Variable } & $n(\%)$ \\
\hline Sexo & \\
Femenino & $647(40,6)$ \\
Masculino & $947(59,4)$ \\
Años de educación & \\
Técnico superior & $398(25,1)$ \\
Educación secundaria (completa o no) & $1190(74,9)$ \\
Índice de masa corporal & \\
Bajo peso & $57(3,6)$ \\
Eutrófico & $821(51,5)$ \\
Sobrepeso & $418(26,2)$ \\
Obeso & $298(18,7)$ \\
Hipertensión arterial & \\
No & $599(37,7)$ \\
Sí & $988(62,3)$ \\
Diabetes mellitus 2 & \\
No & $1303(82,5)$ \\
Sí & $276(17,5)$ \\
Depresión & \\
No & $1220(77,4)$ \\
Nó & $356(22,6)$ \\
\hline Sí & \\
\hline
\end{tabular}

* Los valores absolutos pueden no sumar 1594 debido a la falta de datos en algunas variables

De acuerdo al IMC, encontramos a $418(26,2 \%)$ participantes con sobrepeso y $298(18,7 \%)$ con obesidad. La mayoría de las participantes padecía hipertensión arterial (62,3\%), en menor proporción diabetes mellitus $2(17,5 \%)$, depresión $(22,6 \%)$ y enfermedad cerebrovascular $(4,4 \%)$. De acuerdo a las valoraciones del IMC, se halló que la tasa de prevalencia de bajo peso fue de $24,6 \%$ entre los participantes en el estudio. 
En cuanto al análisis bivariado, se halló que el promedio de puntaje de MoCA fue significativamente mayor en los participantes con sobrepeso, seguido por los obesos y los eutróficos, todos en comparación con los participantes de bajo peso. Aquellos que contaban con un mejor puntaje de MMSE fueron los que tenían sobrepeso, luego obesidad, eutróficos y por último aquellos con bajo peso. Esto indica aquellas que personas con bajo peso presentaron un puntaje MoCA y MMSE más bajo. En cuanto a los puntajes obtenidos en el cuestionario de Pfeiffer, se encontró que el puntaje promedio alto fue significativo principalmente para la categoría de bajo peso y obesidad, seguido de aquellos eutróficos y con sobrepeso. Es decir, aquellos con bajo peso presentaron un puntaje del Cuestionario de Pfeiffer mayor (Tabla 2).

Tabla 2. Análisis bivariado de la muestra del estudio $(n=1594)$

\begin{tabular}{lcc}
\multicolumn{1}{c}{ Variable } & Valor & Valor de $p$ \\
\hline $\begin{array}{l}\text { Cuestionario de Pfeiffer [Me (RIC)] } \\
\text { Bajo peso }\end{array}$ & $4,5(6,3)$ & \\
Eutrófico & $4(3,7)$ & \\
Sobrepeso & $3(2,6)$ & \\
Obesidad & $4,5(2,6)$ & \\
MoCA (x $\pm \mathrm{DE})$ & & $<0,001^{\dagger}$ \\
Bajo peso & $16,2 \pm 8,2$ & \\
Eutrófico & $20,9 \pm 4,6$ \\
Sobrepeso & $21,5 \pm 4,3$ \\
Obesidad & $21,4 \pm 3,6$ \\
MMSE (x \pm DE) & \\
Bajo peso & $13,7 \pm 8,2$ & \\
Eutrófico & $18,8 \pm 4,5$ & \\
Sobrepeso & $19,67 \pm 4,6$ & \\
Obesidad & $19,5 \pm 3,7$ & \\
\hline
\end{tabular}

$x \pm D E:$ media \pm desviación estándar; $M e(R I C)$ : mediana (rango intercuartílico); MoCA: Montreal Cognitive Assessment; MMSE: Mini-Mental State Examination. * Prueba de Kruskall Wallis.

† Prueba Anova de una vía.

Fuente: Elaborado con datos del estudio

Según modelos ajustados por hipertensión arterial, diabetes mellitus, depresión, enfermedad cerebrovascular, educación, edad y sexo, se halló que los participantes con bajo peso tenían valores de escalas cognitivas en rangos más patológicos que los participantes con peso normal, sobrepeso y obesidad (Tabla 3).

\section{DISCUSIÓN}

Nuestro objetivo fue determinar la asociación entre el estado nutricional según IMC y deterioro cognitivo. Encontramos que la cuarta parte de los sujetos ancianos presentaban un estado de malnutrición (bajo peso), porcentaje mucho mayor a lo reportado por Dosil et al., quienes evaluaron, según el mismo estándar de medición, a ancianos mayores de 65 años y encontraron una frecuencia de bajo peso del de $10,8 \%{ }^{[10]}$. Adicionalmente, un estudio realizado por Mantzorou et al., utilizando la escala Mini Nutritional Assessment -que incluye la medición de IMC-, encontró que el $11,3 \%$ de las personas mayores eran desnutridas y el $35 \%$ estaba en riesgo de desnutrición ${ }^{[26]}$. Recientemente, Avendaño estimó que el $18,6 \%$ de un grupo de adultos mayores padecía de desnutrición ${ }^{[9]}$, mientras que Malara et al. encontraron que el 30\% de un grupo de pacientes ancianos tenía desnutrición ${ }^{[11]}$.

A nivel nacional, si bien Guevara identificó que el 10\% de los adultos mayores presentaba desnutrición ${ }^{[12]}$, Córdoba et al. encontraron que la frecuencia de desnutrición era mucho mayor (64,3\%) en ancianos residentes en casas de reposo ${ }^{[27]}$. Las diferencias entre los resultados se deberían a las notables diferencias metodológicas sobre la evaluación del estado nutricional y procedencia de la muestra de estudio.

En cuanto a su relación con el grado de deterioro cognitivo -ya sea mediante MoCA, MMSE o el cuestionario de Pfeiffer- se observó relación significativa entre el bajo peso y presencia de deterioro cognitivo. Estos resultados son similares a otros estudios. Dosil et al., en un estudio realizado en 133 sujetos ancianos en Galicia, encontraron que el IMC se relacionó con el estado cognitivo evaluado por MMSE, y observaron, además, que mientras el IMC disminuía el deterioro cognitivo aumentaba ${ }^{[10]}$. Un estudio realizado entre 174 internos de un hogar de ancianos en Calabria-Italia por Malara et al. apunta también hacia la misma conclusión, puesto que encontraron una fuerte correlación entre el deterioro cognitivo evaluado por MMSE y el empeoramiento del estado nutricional ${ }^{[11]}$. Otros estudios como el de Avendaño ${ }^{[9]}$

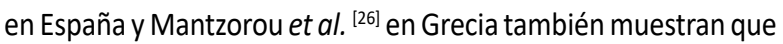
el estado de desnutrición se asoció directamente con el deterioro cognitivo evaluado por MMSE.

Por último, en el estudio se observó que los adultos mayores con sobrepeso y obesidad presentaron un puntaje MOCA y MMSE más alto en comparación a aquellos con bajo peso. Esto se debería a que los primeros suelen estar predominantemente en zonas urbanas; además, tal como lo menciona Álvarez-Dongo et al. ${ }^{[28]}$ esta característica aumenta a medida que disminuye el nivel de pobreza. Con ello se puede inferir que los adultos mayores con obesidad y sobrepeso poseerían un mayor nivel de instrucción y un mejor estado socioeconómico, por lo que no tendrían dificultades para la adquisición, preparación o ingesta de los alimentos.

El presente estudio tiene como fortaleza el presentar datos con base al registro de información de pacientes adultos mayores durante cinco años consecutivos, registrada por médicos especialistas en geriatría. No obstante, existen algunas limitaciones: se resalta que el seguimiento se realizó en ancianos que acuden a una institución de salud, lo que puede haber conducido a una estimación alterada de la prevalencia del deterioro nutricional y cognitivo, además de ser un hospital que atiende a militares retirados y sus familiares, lo que podría significar que nuestros resultados no sean extrapolables a la población general.

Otra limitación del estudio es no haber utilizado variables bioquímicas como albúmina y globulina, además de otros 
Tabla 3. Análisis de regresión lineal para determinar asociación entre índice de masa corporal y trastorno neurocognitivo ( $n=1594)$

\begin{tabular}{|c|c|c|c|c|}
\hline Categoría & Coeficiente $\beta$ & Error estándar & Valor de $p$ & IC 95\% \\
\hline \multicolumn{5}{|l|}{ Trastorno neurocognitivo según: } \\
\hline \multicolumn{5}{|l|}{ MoCA (modelo 1)* } \\
\hline Eutrófico & 3,9 & 0,6 & 0,001 & 2,9 a 5,1 \\
\hline Sobrepeso & 4,8 & 0,6 & 0,001 & 3,7 a 6,0 \\
\hline Obesidad & 4,6 & 0,6 & 0,001 & 3,4 a 5,8 \\
\hline \multicolumn{5}{|l|}{ MMSE (modelo 2)* } \\
\hline Eutrófico & 4,4 & 0,6 & 0,001 & 3,3 a 5,6 \\
\hline Sobrepeso & 5,5 & 0,6 & 0,001 & 4,2 a 6,7 \\
\hline Obesidad & 5,2 & 0,6 & 0,001 & 3,9 a 6,4 \\
\hline \multicolumn{5}{|c|}{ Cuestionario de Pfeiffer (modelo 3)* } \\
\hline Eutrófico & $-0,5$ & 0,4 & 0,176 & $-1,2$ a 0,22 \\
\hline Sobrepeso & $-1,5$ & 0,4 & 0,001 & $-2,3$ a $-0,8$ \\
\hline Obesidad & $-0,8$ & 0,4 & 0,043 & $-1,6$ a $-0,1$ \\
\hline
\end{tabular}

IC 95\%: interval de confianza al 95\%; MoCA: Montreal Cognitive Assessment; MMSE: Mini-Mental State Examination.

* Los tres modelos fueron ajustados por hipertensión arterial, diabetes mellitus, depresión, enfermedad cerebrovascular, educación, edad, sexo. En los tres modelos, la categoría de referencia fue "bajo peso".

Fuente: elaborado con datos del estudio

parámetros antropométricos como la medición de circunferencia braquial, circunferencia de la pantorrilla, o la utilización del test Mini Nutritional Assesment para poder evaluar de forma más precisa la malnutrición. En su reemplazo se usó de IMC como parámetro para evaluar el estado nutricional en el adulto mayor, ya que su representatividad como parámetro biométrico varía según la edad debido a cambios en la composición corporal a lo largo del ciclo de vida. Además, las evaluaciones cognitivas utilizadas no son diagnósticas, solo de tamizaje. Sin embargo, nuestros hallazgos respaldan la evidencia científica actual y aportan información importante sobre la asociación entre variables nutricionales y cognitivas.

Concluimos que tener bajo peso se asocia con peor desempeño cognitivo independientemente del instrumento usado, según modelos ajustados por hipertensión arterial, diabetes mellitus, depresión, enfermedad cerebrovascular, educación, edad y sexo. Estos resultados sugieren que es necesario establecer estrategias o programas de intervención para mejorar la nutrición de la población adulta mayor, ya que la prevalencia de deterioro cognitivo aumentaría en aquellos con bajo peso. Se recomienda también se apliquen herramientas de tamizaje como MoCA, MMSE y Pfeiffer en la práctica clínica geriátrica de rutina para abordar estos problemas de salud de manera oportuna y eficaz.

Contribuciones de autoría: Los autores indican haber participado durante la formulación del problema, la recolección de los datos, el análisis de los mismos, la redacción de este artículo y la aprobación de la versión final.

Fuentes de financiamiento: Autofinanciado.

Conflictos de interés: Los participantes niegan conflictos de interés.

\section{REFERENCIAS BIBLIOGRÁFICAS}

1. Blazer DG, Yaffe K, Karlawish J. Cognitive aging: a report from the Institute of Medicine. JAMA. 2015;313(21):2121-2. doi: 10.1001/ jama.2015.4380

2. Instituto Nacional de Estadística e Informática (INEI). Estado de la población peruana 2015. Informe sociodemográfico [internet]. Lima, Perú: INEI; 2015 [citado 10 dic 2018]. Disponible en: http:// www.inei.gob.pe/media/MenuRecursivo/publicaciones_digitales/ Est/Lib1251/Libro.pdf

3. Borrás B, Viña R. Neurofisiología y envejecimiento. Concepto y bases fisiopatológicas del deterioro cognitivo. Rev Esp Geriatr Gerontol. 2016;51(Suppl 1):s3-s6. doi: 10.1016/S0211-139X(16)30136-6

4. Whitley E, Deary I, Ritchie S, Batty G, Kumari M, Benzeval M. Variations in cognitive abilities across the life course: crosssectional evidence from Understandin Society: The UK Household Longitudinal Study. Intelligence. 2016;59:39-50. doi: 0.1016/j. intell.2016.07.001

5. Guyonnet $\mathrm{S}$, Rolland $\mathrm{Y}$. Screening for malnutrition in older people. Clin Geriatr Med. 2015;31(3):429-37. doi: 10.1016/j. cger.2015.04.009

6. López-Contreras MJ, Torralba C, Zamora S, Pérez-Llamas F. Nutrition and prevalence of undernutrition assessed by different diagnostic criteria in nursing homes for elderly people. J Hum Nutr Diet. 2012;25(3):239-46. doi: 10.1111/j.1365-277X.2012.01237.x

7. Osuna-Padilla IA, Verdugo-Hernandez S, Leal-Escobar G, OsunaRamirez I. Estado nutricional en adultos mayores mexicanos: estudio comparativo entre grupos con distinta asistencia social. Rev Esp Nutr Hum Diet. 2015;19(1):12-20. doi: 10.14306/ renhyd.19.1.119

8. Montejano R, Ferrer R, Clemente G, Martínez-Alzamora N, Sanjuan A, Ferrer E. Factores asociados al riesgo nutricional en adultos mayores autónomos no institucionalizados. Nutr. Hosp. 2014;30(4):858-69. doi: 10.3305/nh.2014.30.4.7829

9. Avendaño O. Evaluación del estado nutricional y el deterioro cognitivo en una cohorte de ancianos [tesis doctoral]. Madrid, España: Universidad Complutense de Madrid; 2017. 
10. Dosil A, Dosil C, Leal C, Neto S. Estado nutricional de ancianos con deterioro cognitivo. INFAD. 2013;1(2):297-310.

11. Malara A, Sgró G, Caruso C, Ceravolo F, Curinga G, Renda G, et al. Relationship between cognitive impairment and nutritional assessment on functional status in Calabrian long-term-care. Clin Interv Aging. 2014;9(1):105-10. doi: 10.2147/CIA.S54611

12. Guevara N. Estado nutricional y su relación con el estado cognitivo del adulto mayor en el Club Municipal de Mariano Melgar, Arequipa 2016 [tesis de licenciatura]. Arequipa, Perú: Universidad Nacional de San Agustín; 2017.

13. Sosa M. Deterioro cognitivo en la vejez ¿Fenómeno normal? [trabajo de grado]. Montevideo, Uruguay: Universidad de la República; 2016.

14. Rosenzweig A. Montreal Cognitive Assessment (MoCA) Test scoring and accuracy [internet]. New York, Estados Unidos: Verywellhealth; c2018 [citado 10 dic 2018]. Disponible en: https://web.archive. org/web/20180816182212/https://www.verywellhealth.com/ alzheimers-and-montreal-cognitive-assessment-moca-98617

15. Pedraza O, Salazar A, Sierra F, Soler D, Castillo P, Hernandez A, et al. Confiabilidad, validez de criterio y discriminante del Montreal Cognitive Assessment (MoCA) test, en un grupo de adultos de Bogotá. Acta Med Colomb. 2016;41(4):221-8.

16. Trzepacz P, Hochstetler H, Wang S, Walker B, Saykin A. Relationship between the Montreal Cognitive Assessment and Mini-mental State Examination for assessment of mild cognitive impairment in older adults. BMC Geriatr. 2015;15:107. doi: 10.1186/s12877-015-0103-3

17. Villarejo A, Puertas V. Utilidad de los test breves en el cribado de demencia. Neurología. 2011;26(7):425-433. doi: 10.1016/j. nrl.2010.12.002

18. Kaur N, Belchior P, Gelinas I, Bier N. Critical appraisal of questionnaires to assess functional impairment in individuals with mild cognitive impairment. Int Psychogeriatr. 2016;28(9):1425-39. doi: 10.1017/S104161021600017X

19. Field L, Hand R. Differentiating malnutrition screening and assessment: a nutrition care process perspective. J Acad Nutr Diet. 2015;115(5):824-8. doi: 10.1016/j.jand.2014.11.010
20. Runzer-Colmenares FM, Samper-Ternent R, Al Snih S, Ottenbacher $\mathrm{K}$, Parodi JF, Wong R. Prevalence and factors associated with frailty among Peruvian older adults. Arch Gerontol Geriatr. 2014;58(1):6973. doi: 10.1016/j.archger.2013.07.005

21. Cálculo IMC [internet]. Madrid, España: Sociedad Española para el Estudio de la Obesidad; 2018 [citado 27 set 2018]. Disponible en: https://www.seedo.es/index.php/imc

22. Conroy-Ferreccio G. Sesgos en la medición del índice de masa corporal en adultos mayores. Nutr Hosp. 2017;34(1):251. doi: 10.20960/nh.1002

23. Vogel S, Banks S, Cummings J, Miller J. Concordance of the Montreal cognitive assessment with standard neuropsychological measures. Alzheimers Dement (Amst). 2015;1(3):289-94. doi: 10.1016/j. dadm.2015.05.002

24. Alegría J. Utilidad de la evaluación cognitiva de Montreal en relación al minimental test para determinar deterioro cognitivo en pacientes con enfermedad de Parkinson, Hospital escuela Antonio Lenin Fonseca, enero 2016 [tesis especialista en Medicina Interna]. Managua: Universidad Nacional Autónoma de Nicaragua; 2016.

25. Consejería de Salud. Cribado de deterioro cognitivo - Test de Pfeiffer versión española. [internet]. Andalucía, España: Servicio Andaluz de Salud; [citado 28 set 2018]. Disponible en: https://www.hvn.es/ enfermeria/ficheros/test_de_pfeiffer_version_espanola.pdf

26. Mantzorou M, Vadikolias K, Pavlidou E, Serdari A, Vasios G, Tryfonos $C$, et al. Nutritional status is associated with the degree of cognitive impairment and depressive symptoms in a Greek elderly population. Nutr Neurosci. 2018;1-9. doi: 10.1080/1028415X.2018.1486940

27. Córdova J, Villanueva C. Fuerza de asociación entre el estado nutricional y el deterioro cognitivo en el adulto mayor que reside en casas de reposo en el distrito de San Borja, 2017 [tesis de licenciatura]. Lima, Perú: Universidad Católica Sedes Sapientiae; 2017.

28. Álvarez-Dongo D, Sánchez-Abanto J, Gómez-Guizado G, TarquiMamani C. Sobrepeso y obesidad: prevalencia y determinantes sociales del exceso de peso en la población peruana (2009-2010). Rev Peru Med Exp Salud Publica. 2012;29(3):303-13.

\title{
Las ediciones anteriores de Acta Médica Peruana están disponibles en:
}

\author{
wWW.redalyc.org
}

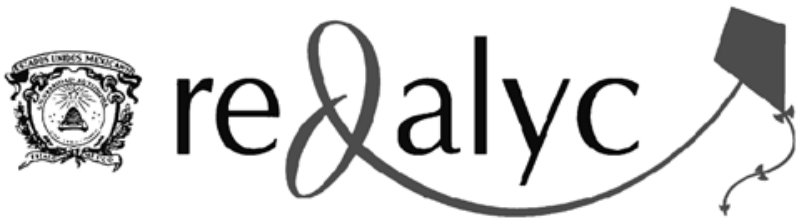

